\title{
ESTUDIO DE LA EVIDENCIA DE CONCEPTO DE GRUPO EN E MODELO DE ADAPTACIÓN DE ROY
}

\author{
María Vilaní Cavalcante G uedes
}

Enfermera, D octoranda del Programa de Pos-graduación en Enfermería de la U niversidad Federal de Ceará, Profesora del Curso de Enfermería. Marcos Venícios de 0 liveira Lopes

Enfermero, Doctor en Enfermería, Profesor Adjunto del Departamento de Enfermería.

\section{Thelma Leite de Araujo}

Enfermera, D octora en Enfermería, Profesora Adjunto del Departamento de Enfermería.

\section{Universidad Federal de Ceará, Fortaleza-Ceará, Brasil.}

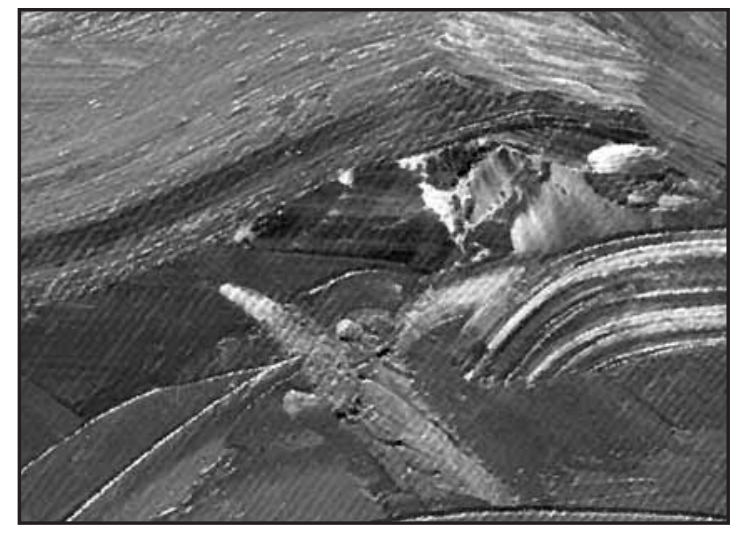

STUDYING THE EVIDENCE IN THE CONCEPT OF GROUP IN ROY'S ADAPTATION MODEL

\section{SUMMARY}

卫

The aim of our study has been the evaluation of the concept of 'group' in Roy's Adaptation Model, taking as basis the inner criterium for evidence suggested by Barnum (1998) in her theoretical analysis pattern. The concept of 'group' is here debated, according to various authors as well as that described by Roy in her different adaptation ways. Our position is that the concept of 'group' in the diverse adaptation models has been adversely affected and that a better description of the Theoretical Model studied should be done especially in the functions of 'role' and 'inter-dependency'.

Key words: Nursing theory, adaptation, group.

\section{RESUMEN}

Q 1 estudio tiene como objetivo evaluar el $\checkmark$ concepto de grupo en el Modelo de Adaptación de Roy tomando por parámetro el criterio interno de evidencia del modelo de análisis de teoría propuesto por Barnum (1998). Discutimos el concepto de grupo con base en diferentes autores, y también según descrito por Roy en sus diversos modos de adaptación. Encontramos que la evidencia del concepto grupo, en los modos de adaptación, se quedó perjudicada y que el Modelo Teórico estudiado necesita una mejor descripción del concepto, sobretodo, en los modos función de papel e interdependencia.

Palabras-clave: Teoría de enfermería, Adaptación, Grupo.

\section{INTRODUCCIÓN}

Ciencias particulares como la Enfermería han buscado sustentación a través de la pesquisa para la ampliación del conocimiento. Al procurar establecer el estatuto de ciencia, elabora teorías que den cuenta de la realidad de su práctica. El ejercicio de la reflexión es complejo, y exige de aquél que se dedica a la actividad, seguir un ritual, un camino que envuelve un ir y venir, garantizando caracterizar el objeto de estudio, de tal forma que los fundamentos que dan sustentación atiendan a las especificidades de lo hacer.

Las teóricas de enfermería han hecho este ejercicio, y las teorías construidas muestran que muy 
aún tendremos por el frente para que alcancemos respuestas que, aunque provisorias, son puntos de partida para experimentos y discusiones con vistas al perfeccionamiento de cada propuesta. Paralelo a esto, una constante revisión de los modelos teóricos ha sido efectuada. Modelos de análisis de las teorías tienen sido desarrollados y el análisis crítico se ha presentado como actual y necesario.

Entre los varios modelos de análisis de teorías existentes, destacamos lo de Barnum (1998). En su propuesta de análisis, la autora presenta la naturaleza del juicio a través de criterios que pueden ser externos e internos. Los criterios externos conciernen al modo como la teoría se relaciona con el mundo real de los hombres, enfermería y salud y son: convergencia con la realidad, utilidad, significación y capacidad de discriminación. Por otro lado, los criterios internos conciernen al juicio interno de la construcción de la teoría y la autora especifica los siguientes: evidencia, consistencia, adecuación y desarrollo lógico.

El criterio de evidencia requiere que una teoría o concepto sea presentado de una forma que pueda ser más bien comprendida por el lector. El autor debe estar atento para el uso de expresiones denotativas y connotativas para no generar ambigüedades, que de cierto modo dificultan el entendimiento y el juicio de la teoría. A evidencia no está asociada a la simplicidad o complejidad de desarrollo de una teoría.

En este sentido, se espera que conceptos y modos trabajados en la teoría sean entendidos de la misma manera por todos los interesados, para que pueda ser implementada, y se llegue a los mismos resultados, o por lo menos, a resultados aproximados, considerando las diversidades culturales.

Entre los conceptos que han sido más recientemente estudiados en la enfermería se destaca lo de grupo, justificado por la importancia que muchos enfermeros atribuyen a la evaluación e implementación de intervenciones en grupos teniendo como base diversos estudios sobre lo tema.

Zimerman (1997a) considera el hombre como ser gregario y que vive en función de sus interrelaciones en grupo. En Abbagnano (1998) encontramos grupo en su significado sociológico como un conjunto de personas caracterizadas por una actitud común o recurrente. En las Ciencias Sociales, grupo significa un conjunto de personas, pero siempre es preciso distinguir entre grupos estructurados y no-estructurados. Las características de los grupos incluyen: relaciones interdependientes entre sus miembros y compartimiento de una misma ideología. Algunos grupos se adaptan a esos criterios tales como: familias, círculos de amistad, clubes de políticos, laborales, pedagógicos entre otros (Silva, 1987).

Existen diversas clasificaciones de grupo. Zimerman (1997b) propone una con base en el criterio de las finalidades que el grupo tiene y presenta dos grandes ramos: grupos operativos y grupos psicoterápicos. Cada un tiene subdivisiones y en la subdivisión de grupos psicoterápicos, encontramos el de la teoría sistémica en la cual, los grupos funcionan como sistemas en constante interacción, complementación y suplemento. Entiende aunque, cualquier modificación en sus elementos afectará el grupo como un todo.

Sabemos que el Modelo de la Adaptación de Callista Roy es una teoría sistémica que incluye el concepto de grupo. Mientras, estudios sobre teorías de enfermería aún son escasos, sobretodo enfocando tal concepto. En este contexto, identificamos la teoría de la adaptación siendo utilizada como base de discusión en algunos estudios, sin embargo, no encontramos referencia al concepto de grupo en los mismos (Lopes, 1998; Lopes \& Araújo, 1999).

Roy, al discurrir en su propuesta teórica, sobre los modos de adaptación de su teoría, se reporta a grupo y hace señas para comunidad y sociedad dis-

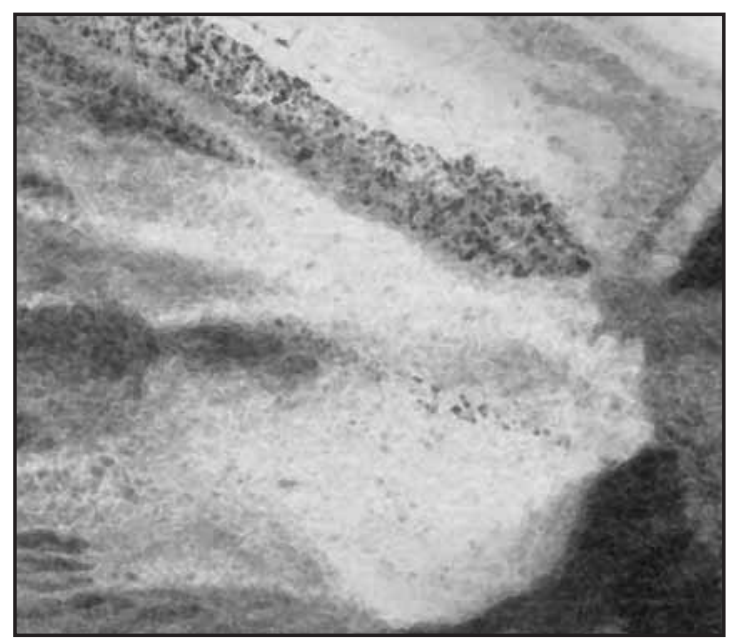


tinguiéndolos con relación al individual. Esta distinción se pone clara cuando se definen los modos de adaptación que reciben denominaciones diferentes cuando son utilizados para evaluar comportamientos de individuos o de comunidad y sociedad. El modo de adaptación fisiológico que se refiere a individuo, pasa a ser físico cuando se refiere a grupos y comunidades. El modo auto concepto es denominado de identidad del grupo. Los modos función de papel y de interdependencia reciben la misma denominación (Roy \& Andrews, 1999).

Con base en el expuesto, objetivamos analizar a evidencia del concepto grupo presentado en la Teoría de la Adaptación de Roy teniendo como base el modelo de análisis de teorías de Barnum (1998).

\section{MATERIAL Y MÉTODO}

Se trata de un estudio teórico reflexivo teniendo como base el concepto Grupo presentado en el Modelo de la Adaptación de Roy. El análisis incluye explanación sobre los principales conceptos del modelo teórico en cuestión y la descripción de la relación entre éstos y el concepto Grupo. Para evaluación del criterio de evidencia se consideró la caracterización presentada por la teórica en las diversas partes de su obra principal investigando la precisión y uniformidad con que el concepto es presentado en las diversas partes del modelo.

La primera etapa del estudio incluyó una revisión teórica que, según Broome (2000), resulta en la identificación y descripción de un nuevo concepto o teoría, o en el refinamiento de uno ya existente. Como base para evaluación se utilizó la última publicación disponible del Modelo de la Adaptación de Roy (Roy \& Andrews, 1999). Paralelo a la descripción son presentados comentarios acerca de la evidencia del concepto Grupo en cada una de las partes descritas. El análisis no fue subdividida para mantener el carácter de continuidad del concepto, mientras la descripción sigue el orden presentado por la autora. Se inicia con los conceptos de base del Modelo pasando por cada uno de los modos de adaptación. A pesar del modelo teórico presentar los Modos FísicoFisiológico, Auto concepto-Identidad de Grupo, Función de Papel e Interdependencia, restringimos el análisis a los modos Físico, Identidad de Grupo, Función de Papel e Interdependencia teniendo en vista que éstos presentan definiciones y descripciones específicas para grupos. Los otros dos modos, conforme ya comentado, se limitan a la descripción de aspectos del individuo, fugándose al objetivo del trabajo.

\section{RESULTADOS Y DISCUSIÓN}

Sister Callista Roy idealizadora de la teoría de la Adaptación inició su trabajo bajo la orientación de Dorothy E. Johnson de 1963 a 1966. La continuidad regular de su estudio resultó en una vasta publicación, lo que demuestra preocupación de la autora con el perfeccionamiento de su teoría (Roy, 1988; Roy, 1993; Roy, 1995; Roy, 1997; Roy, 1999; Roy, 2000).

Roy considera adaptación como proceso y resultado pensado y sentido por la persona como individuo o grupo expuesto a estímulos que pueden ser de diferentes naturalezas. Sería, entonces, el proceso y resultado por medio de lo cual las personas pensando y sintiéndose, como individuos o grupos, se vuelven conscientes y optan por crear una integración humana y ambiental (Key Definitions, 2003).

Mientras grupo, el sistema de adaptación está subdividido en cuatro modos: Físico, Identidad de Grupo, Función de Papel e Interdependencia. El modo físico de adaptación de grupos está descrito como la manera en la cual el sistema humano colectivo de adaptación manifiesta la adaptación relativa a los recursos operativos básicos, participantes, facilidades físicas y recursos fiscales (Roy \& Andrews, 1999).

Los participantes en el modo físico sano las personas que componen el grupo y, como tales, deben vivir en sintonía con los intereses del grupo buscando establecer mecanismos que faciliten la adaptación. Es válido decir que las delimitaciones territoriales no deben ser los definidores de un grupo, porque son determinadas por los propios participantes. Los componentes del grupo deben tener capacidades, aptitudes, conocimientos y otros atributos que puedan contribuir para mantener la entidad que da sustentación al grupo.

Para la perecuación del proceso de adaptación en grupo, Roy refiere que las facilidades físicas, 


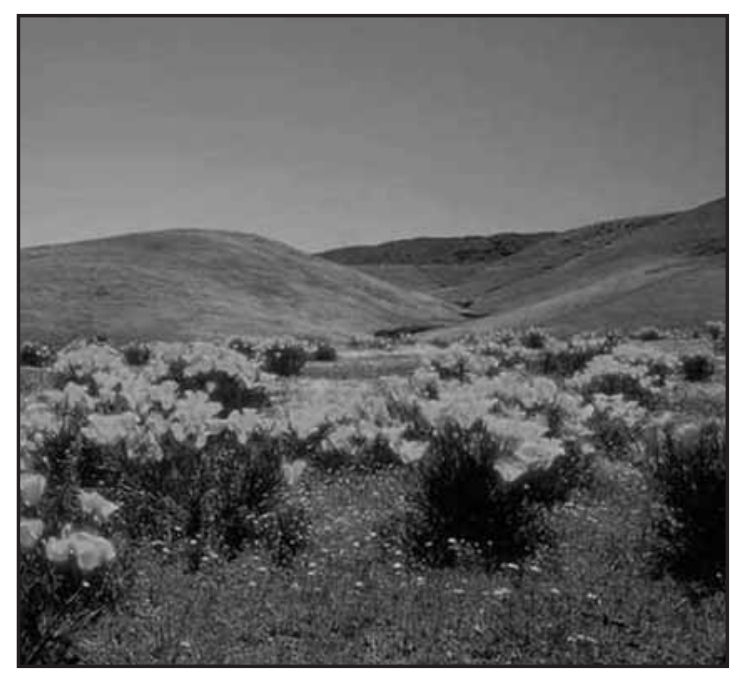

con ambientes diversos, desde aquellos destinados la acomodación de las familias, ambientes para reunir el grupo e incluso el espacio físico para abrigar la organización, son imprescindibles para el buen desempeño del grupo. Aquí también están incluidos recursos financieros que el grupo dispone para su mantenimiento.

A pesar de describir los recursos financieros como punto importante para el modo físico, tal preocupación aparece de modo apocado en la teoría. La autora afirma que se deudas inadecuadas persisten por largo tiempo, la integridad de la totalidad del sistema es comprometida, resultado de comportamientos ineficaces.

En el modelo no son presentadas definiciones y características de los grupos que presentan la estructuración física que las autoras determinan. Grupos ocasionales o formados en antesalas de consultorios, que son frecuentemente utilizados para sesiones de educación en salud, no presentan tal estructuración.

El modo de identidad de grupo es caracterizado por la manera como las personas, en grupos, se perciben basadas en el retorno dado por el grupo / ambiente. Este modo incluye las relaciones interpersonales, auto-imagen de grupo, ambiente social, y cultura.

Para la identidad del grupo es necesario integración compartida de relaciones, metas, valores y co-responsabilidad para la realización de metas; honestidad; solidez; habilidades de relación en grupo y otros atributos que identifican el grupo.
Las autoras reconocen que adaptación en el grupo prevé el compartimiento de relaciones, metas, y valores que crean un ambiente social, y cultivan una auto-imagen de grupo y co-responsabilidad para alcance de esas metas. Por ese punto de vista, los miembros identificados con el grupo tienen sentido de solidaridad, de unión y presentan comportamiento compatible.

En este modo, los procesos comprometedores de la identidad de grupo envuelven disfunción sexual, ansiedad y baja moral. La disfunción sexual, cuando manifestada por un participante, es considerado un proceso comprometedor de la identidad del grupo porque todos participantes deben respetar sus pares y estén unidos por el bienestar general. Personas en estado de ansiedad pueden desestabilizar el grupo como todo. A baja moral se manifiesta a través de la baja disposición de sus miembros para actividades relacionadas a las metas y a lo mantenimiento de las relaciones del grupo.

Algunos aspectos de este componente se presentan sin la debida aclaración de como implementarlos y, en aquellos donde aparece, la explicación es débil y poco convincente. No son trabajadas, de forma apropiada, las definiciones de relaciones interpersonales, auto-imagen de grupo y ambiente social, que son componentes básicos de la identidad de grupo.

El modo función de papeles es definido por Roy \& Andrews (1999) como los papeles que la persona ocupa en la sociedad. Completan diciendo que la necesidad básica asociada al modo de función de papel ha sido identificada como integridad social para individuos y evidencia del papel para grupos o colectividades.

Las autoras aclaran que el modo función de papeles cuando se refiere a grupos, destaca la integridad social y a evidencia del papel como sus necesidades básicas. Resaltan aún, que el modo compuerta los papeles primarios, secundarios y terciarios, que son modificados en el transcurso de la vida, en función de la maduración del grupo desde la estabilidad y respeto alcanzado.

En un grupo, los participantes presentan comportamientos del tipo: instrumental y expresivo de modo que colabore con el desarrollo del grupo. Los comportamientos instrumentales son orienta- 


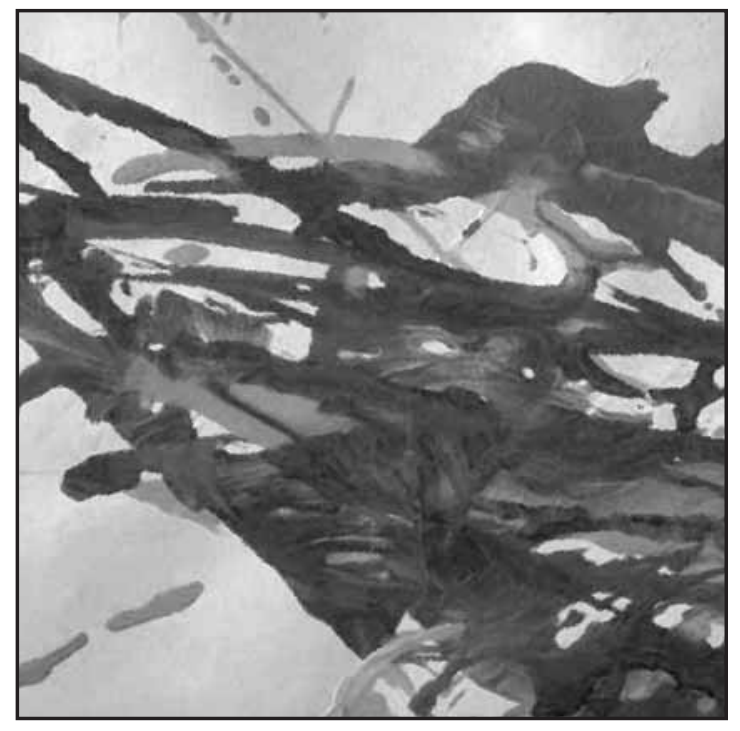

dos a las metas, requiriendo planificación. Mientras que los expresivos son más vueltos para los aspectos afectivos en las relaciones entre los participantes, y envuelven un actuar cuyos resultados pueden aparecer de inmediato.

También en este modo, percibimos fragilidades en la diferenciación cuando se trata de las especificidades de grupos y de individuos. Los ejemplos, dados por las autoras, se quedaron alrededor de grupos muy específicos, como lo de enfermeras, no detallando papeles a ser evaluados en otros grupos como los de pacientes acompañados.

El modo interdependencia es definido por Roy \& Andrews (1999) como las relaciones íntimas de las personas dirigidas a satisfacer necesidades de afecto, desarrollo, y recursos para que alcancen integridad relacional.

En el grupo, la interdependencia se muestra por la necesidad de alcanzar la integridad relacional usando los procesos de desarrollo y adecuación de recursos, a través de aprendizajes y maduración de relaciones creando condiciones para satisfacer las necesidades básicas más elementales con la cooperación de otros.

Las relaciones interdependientes se clasifican en dos categorías: otros significantes, claramente expreso para el individuo, pero no explícito para grupo y, sistemas de soporte que incluyen otras personas, grupos, organizaciones y otros con relación al individuo. En esta categoría también falta evidencia cuando las autoras se reportan a grupo.
Este modo de interdependencia tiene subdivisiones de sus componentes a: el contexto, la infraestructura y el pueblo. El contexto contempla los estímulos internos y externos. Los estímulos externos incluyen aspectos económicos, sociales, políticos, culturales, religiosos entre otros, que son fundamentales para el desarrollo de grupos. Por su lado, los estímulos internos se quedan por cuenta de la misión, visión, principales valores, metas, planos, también imprescindibles para el crecimiento del grupo. La infraestructura cuenta con niveles de adaptación: procesos relacionáis, procesos de desarrollos y procesos de recursos. Las personas en este modo abrigan los componentes: capacidades de enfrentamiento, conocimiento, habilidades, actitudes y comprometimiento que son básicos.

\section{CONSIDERACIONES FINALES}

El estudio que presentamos producido con base en el Modelo de la Adaptación de Roy mostró que, para el concepto de grupo, la teoría no trae a evidencia como criterio interno de una teoría conforme el modelo de análisis utilizado. El concepto de grupo presenta una característica de estructuración y de esta manera, se vuelve difícil su implementación por los enfermeros en la práctica de enfermería, que trabajan diariamente con clientelas las más diferenciadas. Un detalle lindante es el hecho de las autoras no han dejado claro cual la definición de grupo y comunidad y esto se constituyó un factor que dificulta el entendimiento.

La teoría de la adaptación trae una riqueza de detalles, que siendo adaptada a las diversas realidades, traerá grandes contribuciones para la práctica de los enfermeros. Sin embargo, no es posible trasplantarla sin ajustes a nuestro modo de pensar, sentir y actuar. El estudio está lejos de ser concluido. Merece revisiones y refinamientos en la comprensión de las sutilezas que vienen empotradas en el texto. Reconocemos que es un análisis complejo, pero también percibimos qué ni todo cuanto es producido en otra cultura mismo en momento contemporáneo puede ser utilizado sin un análisis más profundizada.

\section{BIBLIOGRAFÍA}

- Abbagnano, N. (1999). Dicionário de Filosofia. Martins Fontes, São Paulo.

- BARNUM, B. S. (1998). Nursing theory: analysis, appli- 
cation, evaluation. Lippincott, Philadelphia.

- Broome, M. E. (2000). Integrative literature reviews for the development of concepts. En Concept Development in Nursing: foundations, techniques and applications (Rodgers, B.L. \& Knafl, K.A.), W.B. Saunders Company, Philadelphia pp. 231-250.

- Key Definitions. (2003). ACRESCENTAR O ENDEREÇO ELETRÔNICO AQUI Capturado 6 de junio de 2003.

- LOPES, M. V. O. (1998). Adaptação física e diagnóstico de enfermagem em mulheres com angina pectoris. Fortaleza (CE). Ed. Universidade Federal do Ceará.

- Lopes, M. V. O., \& AraúJo. T. L. (1999). Adaptación física según Roy: una revisón conceptual. Enfermería Integral, 49(2), 20-24.

- Roy, C. (1988). An explication of the philosophical assumptions of the Roy Adaptation Model. Nursing Science Quarterly, 1(1), 26-34.

- Roy, C., \& Corliss, C. P. (1993). The Roy adaptation model: theoretical update and knowledge for practice. En Patterns of nursing theories in practice (Parker, M.), National League for Nursing, New York pp. 215-229.
- Roy, C. (1995). Developing nursing knowledge: practice issues raised from four philosophical perspectives. Nursing Science Quaterly, 8(5), 79-85.

- Roy, C. (1997). Future of the Roy Model: challenge to redefine adaptation. Nursing Science Quarterly, 10(1), 42-48.

- Roy, C. (1999). State of the art: dissemination and utilization of nursing literature in practice. Biological Research in Nursing, 1(2),147-155.

- Roy, C., \& Andrews, H. E. (1999). The Roy adaptation model. Appleton e Lange, Stamford.

- Roy, C. (2000). A theorist invisions the future and speaks to nursing administrators. Nursing Administration Quarterly, 24(2), 1-12.

- Silva, B. (Coord) (1987). Dicionário de Ciências Sociais. Fundação Getúlio Vargas, Rio de Janeiro.

- Zimerman, D. E. (1997a) Fundamentos teóricos. En Como trabalhamos com grupos (Zimerman, D. E., \& Osório, L. C.), Artes Médicas, Porto Alegre pp. 23-32.

- Zimerman, D. E. (1997b). Fundamentos técnicos. En Como trabalhamos com grupos (Zimerman, D. E., \& Osório, L. C.), Artes Médicas, Porto Alegre pp. 33-40.

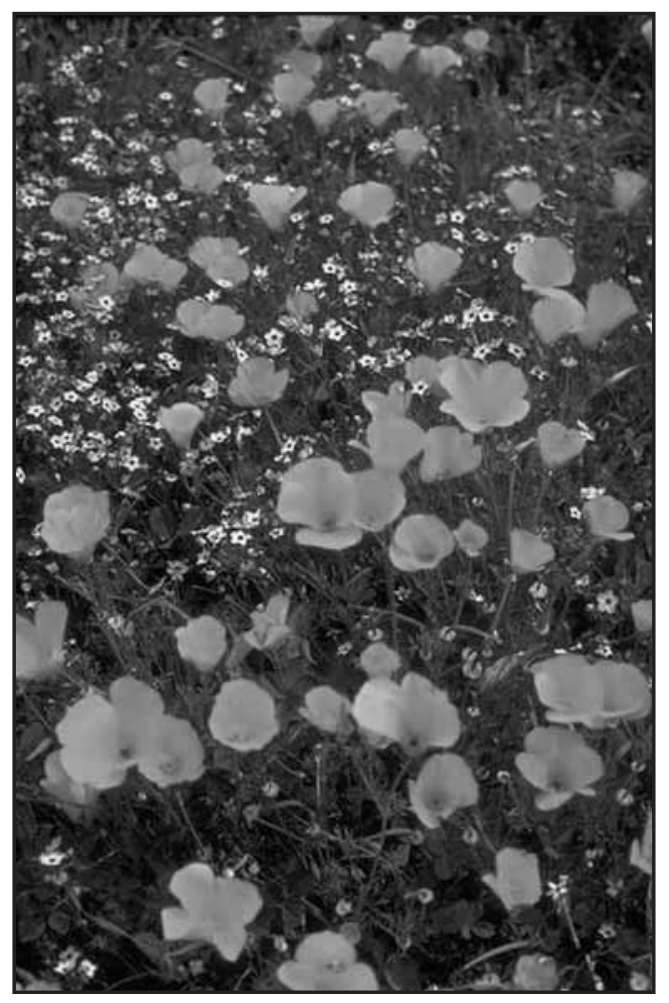

